\title{
ANÁLISE DE MÉTODOS DE REPARO DOS DANOS OCASIONADOS PELA INFILTRAÇÃO POR CAPILARIDADE EM ALVENARIAS
}

\author{
A. L. MARINHO DOS SANTOS \\ Graduanda em Engenharia Civil \\ Universidade de Pernambuco \\ Pernambuco; Brasil \\ adellylorrane@gmail.com
}

\author{
N. B. DE LIMA \\ Mestrando em Engenharia Civil \\ Universidade de Pernambuco \\ Pernambuco; Brasil \\ limanathan7@gmail.com
}

\author{
R. ALMEIDA DE OLIVEIRA \\ Professor Doutor- Engenheiro Civil \\ Universidade Católica de Pernambuco \\ Pernambuco; Brasil \\ romildealmeida@gmail.com
}

\author{
A. MARQUES LOPES ESTOLANO \\ Doutoranda em Engenharia Civil \\ Universidade Federal de Pernambuco \\ Pernambuco; Brasil \\ amandamrqslopes@gmail.com
}

\section{R. ALVES BERENGUER \\ Doutorando em Ciências de Materiais Universidade Federal de Pernambuco \\ Pernambuco; Brasil \\ romildo.berenguer@ufpe.br}

\author{
N. BEZERRA DE LIMA \\ Professora Doutora - Química \\ Universidade Federal de Pernambuco \\ Pernambuco; Brasil \\ nathalia.lima@pq.cnpq.br
}

\section{RESUMO}

A presença da umidade pode comprometer o desempenho mecânico das edificações, exigindo-se um sistema de impermeabilização e estanqueidade eficiente na maioria dos elementos estruturais ou não estruturais. Contudo, uma falha construtiva comum é a não execução de barreiras contra a presença da umidade ou falhas no processo de execução dos métodos ou nas escolhas dos materiais utilizados. As manifestações patológicas desencadeados pela ação da água são facilmente identificadas através de manchas, formação de mofo, fungos e bolor, fissuras, trincas dentre outras, e esses efeitos podem ser minimizados ou eliminados. As infiltrações por capilaridade em alvenarias são dificilmente reversíveis, por se tratar de uma falha ou não existência de impermeabilização dos elementos de fundação. Esse trabalho tem por objetivo analisar os métodos que podem minimizar esses efeitos e garantir uma maior durabilidade das alvenarias e saúde dos usuários da edificação. Através da avaliação de métodos existentes e estudos de caso, buscou-se determinar os procedimentos mais adequados para edificações em fase de utilização. Em todos os casos de umidade em alvenarias analisados neste trabalho, o fator predominante para o surgimento das manifestações patologias foi a ausência de impermeabilização entre a fundação e as alvenarias, sendo a mais recorrente em relação às demais anomalias encontradas.

\section{Palavras-chave: Capilaridade, Impermeabilização, Infiltrações, Manifestações patólogicas.}

\begin{abstract}
The presence of humidity can compromise the performance of buildings, which requiring an efficient waterproofing and tightness system on most related and unrelated elements. In this sense, constructive failures are widely due to the execution of barriers against the presence of capture and failures in the method execution process, as well as in the choices of the materials employed. Pathological manifestations caused by the action of water are easily identified through stains, mold, fungi and mold, fissures or cracks among others, and these effects can be minimized or eliminated. On the other hand, capillary infiltration in masonry is hardly reversible, because it is a failure or there is no waterproofing of the foundation elements. The main goal of the present work is to analyze a set of methods that can change these effects and ensure greater wear and tear on the masonry and health of users of the edition. By assessing existing methods and case studies, the most appropriate procedures for changes in the phase of use were evaluated. Results revealed that for all masonry cases analyzed in this work, the predominant factor for surgery of pathological manifestations was the loss of waterproofing between a foundation and the masonry, being the most recent in relation to the other anomalies employed.
\end{abstract}

Keywords: capillarity, waterproofing, infiltrations, pathogenic manifestations. 


\section{INTRODUÇÃO}

As manifestações patológicas causadas pelas infiltrações em alvenarias podem ser intensificadas pela combinação da ação dos ventos (pressão), direção e intensidade das chuvas e condições de exposições. Além desses fatores, as fissuras são eventuais anomalias que estimulam essa degradação (TAGUCHI, 2010). Para minimizar tais efeitos é necessário um estudo minucioso dos mecanismos de penetração da água nesses elementos, levando em consideração as características dos materiais, os revestimentos utilizados, a configuração arquitetônica e a qualidade de execução (SOUSA, 2002). Para que o fenômeno ocorra segundo Silva e Abrantes (2007), são necessárias três condições simultâneas: a presença de água, a existência de materiais com porosidade "capilar" e a possibilidade de comunicação entre esses dois fatores.

A água que se acumula no solo em contato direto com o elemento não impermeabilizado provoca a redução da vida útil devido a elevada absorção por capilaridade (MENDES, 2011). Além disso, a maioria dos materiais de construção possuem uma capilaridade elevada. A ascensão da água é inversamente proporcional ao diâmetro dos condutos capilares, que são canais que percorrem todo o material através de uma rede de conexões por onde a água passa vencendo a força da gravidade (NAPPI, 2002). De acordo com Lottermann (2013), se esses condutos forem finos o suficiente, combinados com a tensão superficial causada pela coesão entre as moléculas do líquido e a adesão deste à superfície, inicia o processo de ascensão da água através do material poroso.

\section{INFILTRAÇÃO POR CAPILARIDADE}

De acordo com Coimbra (2010), alguns fatores podem ser considerados na análise do comportamento das paredes frente à ação da água da chuva:

- Clima e exposição do local;

- Constituição dos revestimentos;

- Constituição do tosco da parede;

- Configuração arquitetônica da edificação;

- Qualidade de execução.

Os materiais das construções civis, como os blocos cerâmicos (tijolos), argamassas e concretos entre outros. Esses materiais apresentam porosidade consideráveis, sobre o ponto de vista dos materiais macroporosos, mesoporosos e microporosos. Portanto, devem ser protegidos contra a incidência da chuva pelo contato direto ou por ação capilar. Segundo Verçosa (1991), a umidade além de ser uma possível causa das manifestações patológicas é o meio necessário para que grande parte das anomalias nas construções ocorra. Como por exemplo, o surgimento de eflorescência, mofo, fissuras e rachaduras devido a recalques na fundação etc.

Um método que pode ser eficaz para reduzir a ascensão capilar em alvenarias é a ventilação da base das paredes. De acordo com a Figura 1, esse sistema de ventilação, que só pode ser utilizado se a cota inferior for superior ao nível do lençol freático, é formado por canais ventilados naturalmente ou associados a um dispositivo de ventilação mecânico (GUIMARÃES E FREITAS, 2018). 
Figura 1. Esquema do método de ventilação para minimizar o efeito de ascensão capilar

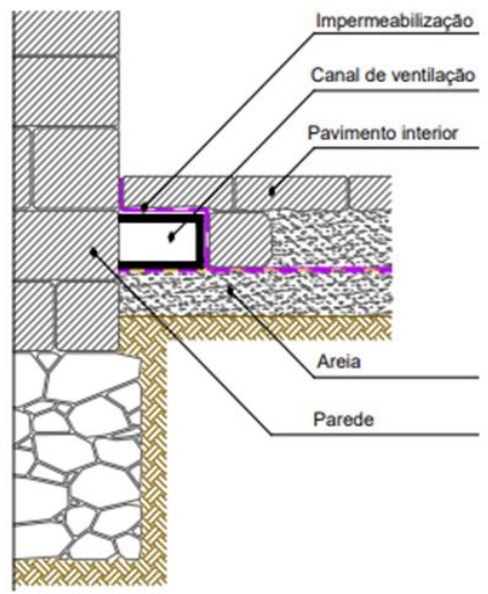

Fonte: Guimarães e Freitas (2018)

Para melhorar a resistência do material sobre o ponto de vista da umidade, é necessário utilizar revestimentos que supram tais requisitos. Podem ser utilizados revestimentos de impermeabilização ou de estanqueidade. Esse último pode, por si só, garantir um bom desempenho do ponto de vista da estanqueidade, mesmo na ocorrência de fissuração (COIMBRA, 2010).

Segundo Bauer (2014), a água pode infiltrar na construção de algumas maneiras. A infiltração pode ocorrer sob pressão, por percolação, ou pelas forças de capilaridade. Esta última ocorre normalmente nas paredes de alvenarias em contato direto com o solo úmido com uma camada de impermeabilização inadequada ou inexistente. Um exemplo comum é o que ocorre nas paredes de alvenaria em contato com o solo úmido sem uma camada impermeabilizadora, uma vez que os blocos cerâmicos (tijolos) são muito porosos, ou seja, apresentam muitos vazios capilares.

\section{ESTUDOS DE CASO}

Através da análise técnica do estado de conservação de duas Sedes gerenciadas pela equipe técnica do Governo de Pernambuco, a Sede A (SDA) e a sede B (SDB), foram identificadas anomalias semelhantes em ambos os casos. Segundo o relatório e as imagens fornecidas, as duas edificações analisadas apresentam avançada deterioração devido a infiltrações nas alvenarias.

De acordo com os dados fornecidos pela equipe técnica, a sede (SDA) foi inaugurada após reformas ajustadas 'para o funcionamento da sede (DAS) no dia 09.11.15, e a inspeção foi realizada no dia 23.08.19, tendo uma idade de aproximadamente 3 anos e 10 meses. Porém o estado atual de deterioração indica erros de projeto e execução, e falta de manutenção preventiva e corretiva, após o aparecimento das primeiras manifestações patologias (HELENE, 2003). Na Figura 2 e 3 podemos identificar infiltrações por capilaridade, que segundo o relatório de inspeção é o principal responsável pela deterioração das alvenarias nessa edificação. 
Figura 2. Infiltração por capilaridade na área externa da sede A (SDA).

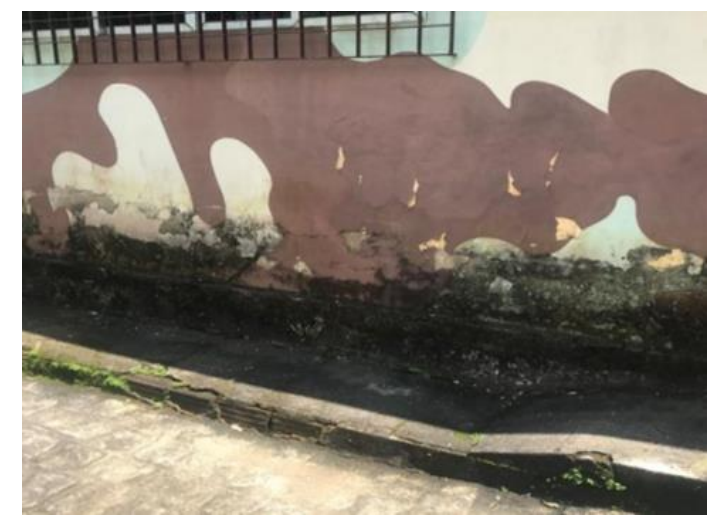

Fonte: Os autores (2019)

Figura 3. Infiltração por capilaridade na área interna da sede A (SDA)

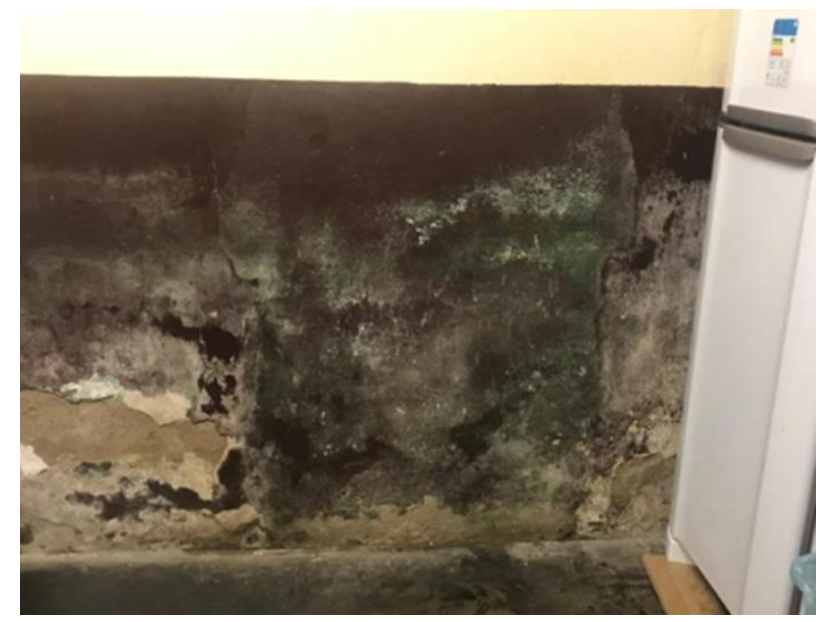

Fonte: Os autores (2019)

O segundo estudo de caso foi a inspeção realizada na sede B (SDB), na cidade de São Lourenço da Mata em Pernambuco. A inspeção foi feita no dia 07.08.19 e a idade da edificação não foi informada. Através da inspeção visual, essa edificação apresentou maior grau de deterioração e incidência das manifestações patologias. A infiltração por capilaridade continua sendo a anomalia mais frequente nos estudos de casos apresentados, e nesse caso principalmente na área externa como mostra a Figura 4. 
Figura 4. Infiltração por capilaridade na área externa da sede B (SDB)

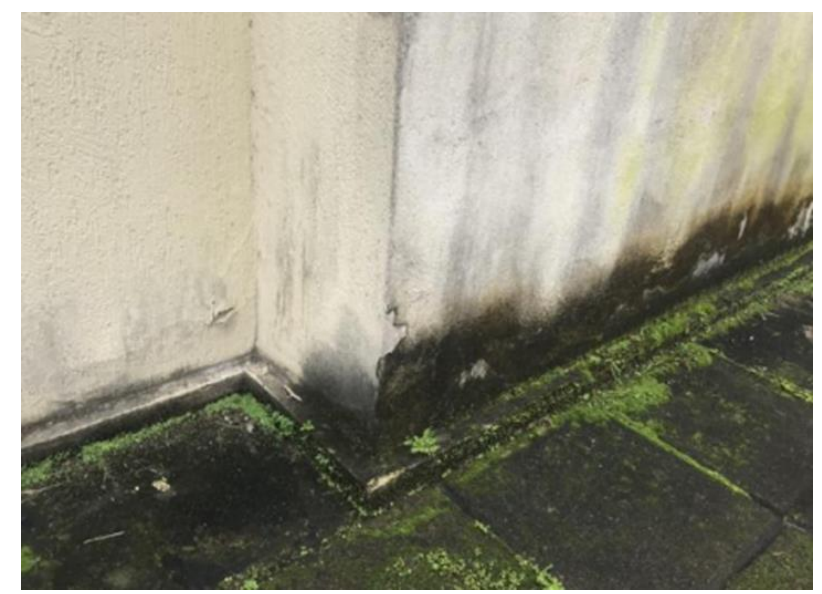

Fonte: Os autores (2019)

Foi constatado que, em ambos os casos, não houve projeto de impermeabilização e que na etapa de execução foi desenvolvida de maneira a desejar conforme anamnese desenvolvida. As patologias geradas pela negligência na fase de projeto foram imediatamente identificadas pelo estado avançado de deterioração do revestimento interno e externo da instituição. As manifestações patológicas como bolor, mofo e desplacamento do revestimento foi identificado em toda a área externa e em todos os cômodos.

O bolor e o mofo, que surgem por intermédio da umidade, causam a deterioração precoce das alvenarias, proporcionando um ambiente insalubre e estéticos. Essas manifestações patológicas surgem com maior incidência em paredes externas, e em paredes internas, devido à baixa ventilação com pouca incidência de ondas eletromagnéticas do Sol (KAUFFEMAN, 2017). Tendo como boa parte das ondas eletromagnéticas que são produzidas pelo Sol, localizadas no intervalo das radiações infravermelhas, luz visível e ultravioleta (KAUFFEMAN, 2017). Nos casos apresentados, há uma intensificação desses efeitos devido a deficiência ou ausência de manutenção corretiva para minimizar a deterioração precoce desses elementos (HELENE, 2003).

As tratativas mais comuns para os casos podem diminuir esses efeitos, mas é improvável a sua eliminação por completa. Isso ocorre devido a deficiência nos métodos de impermeabilização ou a ausência desta. Devido aos métodos e materiais utilizados na execução, o aparecimento das possíveis manifestações patologias ocorrem precocemente e exigem uma manutenção corretiva nos primeiros meses de uso.

Um dos métodos comuns para essas situações seria a eliminação total da umidade devido aos problemas na impermeabilização da alvenaria que pode ser obtida através da injeção de produtos cristalizantes em furos executados na base do local danificado, impedindo a ascensão da umidade (LOTTERMANN, 2013).

Além desse método, a sugestão fornecida pelo laudo técnico seria uma intervenção contundente no sentido de minimizar tais danos, com a retirada da argamassa em no mínimo um metro de altura, ou até onde há manchas características dessa patologia, aplicar a limpeza com uma solução de água com água sanitária de 1:1, que deve ser aplicada quente com hidro jateamento no tijolo aparente. A partir disso, aplicar impermeabilizante no substrato (alvenaria aparente) e logo em seguida aplicar o revestimento. Essa como as outras tratativas não são definitivas. Retardam o surgimento do problema e é necessário que se repita o procedimento assim que a patologia se manifestar novamente.

Outro método sugerido por Silva e Abrantes (2007) é a utilização de reboco hidráulico na fachada para auxiliar na impermeabilização das regiões mais afetadas pela umidade. Para que esse método seja eficiente, é necessário que não exista fissuração superficial. As camadas interiores podem e devem ser mais ricas em cimento para diminuição da porosidade e a tendência de a umidade não migrar para o interior da edificação.

Ações preventivas podem ser tomadas para evitar esses tipos de patologias. Segundo Silva e Abrantes (2007), além da utilização de materiais menos porosos em contato com zonas úmidas, é necessário utilizar barreiras hídricas nos 
elementos de fundação e os que estão em contato com o solo. Essas ações só serão viáveis economicamente no período de projeto e execução,

\section{CONCLUSÕES}

Diante da análise apresentada, é perceptível que em ambos os casos não houve manutenção periódica devido a avançada deterioração dos elementos das edificações. Alguns reparos foram identificados na inspeção, porém nenhum acompanhamento técnico foi realizado antes deste.

A manifestação patológica mais comum identificada, foram as infiltrações nas alvenarias. Isso reflete os erros na execução da fundação associado a ausência de análise do solo. Podemos observar que procedimentos essenciais como impermeabilização, análise do solo e o correto dimensionamento das peças estruturas compromete a vida útil da edificação e podem acelerar a deterioração da estrutura inviabilizando seu funcionamento.

Foi identificado erosão no solo, típico de solos argilosos, e o contato direto da fundação com a água pluvial, intensificando a infiltração direta ou por capilaridade. Além desses pontos analisados, as deformações devido a esse tipo de solo ocasionam o surgimento de trincas e fissuras que facilitam a entrada de agentes externos.

Alguns métodos apresentados para minimizar os efeitos das infiltrações aumentam o custo da manutenção que poderia ser evitado nas fases iniciais de projeto.

\section{REFERÊNCIAS}

TORRES, M. I. Humidades ascencionais. Tese de Doutoramento - Universidade de Coimbra, Coimbra, 2004.

Henriques, Fernando M.A. - "Humidade em paredes" - Edifícios - Série Conservação e Reabilitação, nº, LNEC, Lisboa, 1994.

BAUER, L. A. Falcão. Materiais de construção. Rio de Janeiro, LTC, 2014

VERÇOSA, E.J. Patologia das Edificações. Porto Alegre, Editora Sagra, 1991.172p.

LOTTERMANN, A.F. Patologias em estruturas de concreto: Estudo de caso. Universidade Regional do Noroeste do Estado do Rio Grande do Sul, 2013.

HELENE, Paulo. R. L. (Coord.). Manual de reparo, proteção e reforço de estruturas de concreto. São Paulo: Red Rehabilitar, 2003, p. 370.

COIMBRA, T.L.A. Patologias em Alvenarias de preenchimento induzidas por deformação dos suportes. Dissertação de Mestrado. Faculdade de Engenharia - Universidade do Porto, 2010.

SILVA J.M.; ABRANTES, T.V. Patologias em paredes de alvenaria: Causas e soluções. Seminário sobre Paredes de Alvenaria, P.B. Lourenço et al. (eds.), 2007.

SOUSA, H. Alvenarias em Portugal: Situação atual e perspectivas futuras. Seminário sobre Paredes de Alvenaria, P.B. Lourenço et al. (eds.), 2002.

TAGUCHI, M.K. Avaliação e qualificação das patologias das alvenarias de vedação nas edificações. Dissertação de Mestrado, Universidade Federal do Paraná, 2010.

RIGHI, G.V. Estudos do sistema de impermeabilização: Patologias, prevenções e correções - Análise de casos. Dissertação de Mestrado. Universidade Federal de Santa Maria. 2009. 